\title{
REVIEW OF FACIAL EXPRESSION RECOGNITION SYSTEM AND USED DATASETS
}

\author{
Shyna Dutta ${ }^{1}$, V.B. Baru², \\ ${ }^{1}$ ME Student, Department of Electronics and Telecommunication, Sinhgad College of Engineering Vadgaon, Pune, \\ shainadutta5@gmail.com \\ ${ }^{2}$ Associate Professor, Department of Electronics and Telecommunication, Sinhgad College of Engineering Vadgaon, \\ Pune,vbbaru@gmail.com
}

\begin{abstract}
The human face is main part to recognize the individuals as well as provides the important information, current state of user behavior through their different expressions. Therefore, in biometric area of the research, automatically face \& face expression recognition attracts researcher's interest. The other areas which use such technique are computer science medicine, psychology etc. Usually face recognition system is consisting of many internal tasks. Face detection is thefirst task of such systems. Due to different variations across the human faces, the process of detecting face becomes complex. But with help of different modeling methods, it becomes possible to recognize the face and hence different face expressions. This paperpresents a literature review over the techniques and methods used for facial expression recognition. Also, different facial expression datasets available for the research or testing of existing methods of facial expression recognition are discussed.
\end{abstract}

Keywords: Facial Expression, Face Detection, Features Extraction, Recognition, datasets.

\section{INTRODUCTION}

Now days, the task of face recognition is widely used application of image analysis as well aspattern recognition. This process has been taken the significant attention during the last decade.At least two reasons account for this trend: Wide range of first commercial and law enforcementapplications, and the second after 30 years of research is the availability of viable technologies.Increase and rising security concerns and face recognition of practical life force received a facial recognition and face SatyaHydro system automatically to identify a person or a digital image as much as processing digital, still face picture is carried out to verify a computer application can beconsidered as facial expression recognition [19].The system was introduced in 1978 by Suwa et. Al. creating a facial expression recognition system the main point of face detection and feature extraction and image alignment, normalization, categorization. There are techniques that are usedto identify the facial expression number [2].

In human-to-human conversation, the articulation and perception of facial expressions form a communication channel in addition to voice which carries vital information about the mental, emotional, and even physical state of the persons in conversation. A person's facial expressions in its simplest form is a more subtle happy or angry thoughts, feelings or understanding of the speaker expected or unexpected response from listeners, sympathy, or even what the speaker is saying no signal can provide to computing background, brings our everyday human user to remain atthe forefront in the fabric will move to absorb. This set up a generally prediction, pervasive computing and ambient intelligence such as needed to achieve the next generation ofcomputing [22]. it's easy to naturally occurring multimodal human-human communication focused response to the user interface will need to be developed to identify such interfaces and intentions and as expressed by feelings of social and emotional indicators will need to have the ability. This vision of the future motivates the research for automated recognition of nonverbal actions and expression. Facial expression recognition, computer vision, pattern recognition and human-computer interaction research has attracted increasing attention in communities. Automatic recognition of facial expressions is so affective computing technologies, intelligent tutoring systems, including various forms the essence of the next generation computing equipment, patient monitoring systems, and etc. personal wellness profiled [13]. Human face other gender, different age groups and other physical characteristics of a person varies.

Figure1 below showing the general framework of automatic facial expression recognition. Fromthe figure, there are three important phases of overall system. First is face detection task in which first prominent features are extracted and then face is identified. The second step is where the facial feature extraction and recognition of facial expressions to the overall features are removed at the end of the last step in classification. Facial expressions of input image are then recognized. In this paper, facial expression recognition system is introduced, that is, the various stages of the various methods used for.

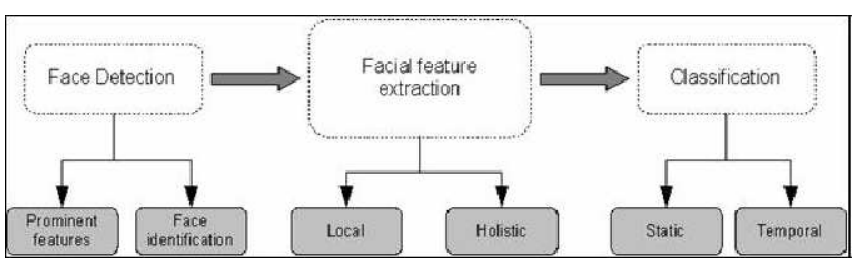


Fig-1: Generalized Framework for Automatic Facial Expression System

\section{LITERATURE REVIEW}

In literature review, many methods for face and facial expression recognition have been presented; however each of them having their own limitations.

In [1], Eigenfaces and in [2], Fisherfaces, which are built on Principal Component Analysis(PCA) [1]; the more recent 2D PCA [3], and Linear Discriminant Analysis [4] are also examples of holistic methods. Although these methods have been studied widely, local descriptors have gained attention because of their robustness to illumination and pose variations.

In [5], Heiseleet al. showed the validity of the componentbased methods, and how they outperform holistic methods. The local-feature methods compute the descriptor from parts of the face, and then gather the information into one descriptor. Among these methods are Local Features Analysis [6], Gabor features [7], Elastic Bunch Graph Matching [8], and Local Binary Pattern (LBP) [9]. The last one is an extension of the LBP feature, that was originally designed for texture description [10], applied to face recognition. LBP achieved better performance than previous methods, thus it gained popularity, and was studied extensively. Newer methods tried to overcome the shortcomings of LBP, like Local Ternary Pattern (LTP) [11], and Local Directional Pattern (LDiP) [12, 13]. The last method encodes the directional information in the neighborhood, instead of the intensity. In [14] [15, Zhang et al. explored the use of higher order local derivatives (LDeP) to produce better results than LBP. Both methods use other information, instead of intensity, to overcome noise and illumination variation problems.

In [20], Peng Yang et al. present a novel approach of facial action units (AU) and expression recognition based on coded dynamical features. In [21], Neeta et al.proposes amethod which is implemented using 2D appearance-based local approach for the extraction of intransient facial features and recognition of four facial expressions.

In [22], Anithaet al. discussed in the paper about the various facial expression databases are available with different variations like illumination, expression, size, shape, color, and texture. In [23], Frank et al. compare performance ration on JAFFE database of facial expression

recognition. In [24], Wai Kin et alconsider 2-D gabor filter to obtain palm print and texture feature extraction for authentication. They describe five modules to get satisfactory results which are Palmprint Acquisition, Preprocessing, Textured Feature Extraction, Matching, and at last Database is usedto store template.

\section{OVERVIEW OF FACIAL EXPRESSION RECOGNITION SYSTEM}

Human facial expression recognition problem of three problem areas (fig.1) also includes: (1)finding faces view, (2) extracting facial features and/or facial features change as the speed of analysis, and some facial expression interpretations categories (for example, emotions, facialmuscle actions to classify this information found in the facial area), (3) `. Face the problem of finding a division problem (machine vision) or (pattern recognition) is a problem in locating, it can be seen as a human face identification of all areas in the view refers to the head in pretend occlusions and variations. Clutter, and Regardless of lighting conditions face (face localization, face detection) should solve the problem of non-rigid movements. Facial expression and facial shape,color \& texture are presence of a high degree of variability in this problem even more difficult. Many techniques to detect faces in still images have been developed. However, most of them only honest face frontal or near frontal view can detect faces arguably the most employed detector automatically. Facial expression analysis in real-time is proposed by Viola and Jones face detector. Feature extraction of problem a dimensionality reduction problem (in machine vision and pattern recognition) as can be seen it referenced that input data to encode features relevant information from a low-to change the input data set represented.

Main problem of the facial feature extraction from input images may be divided into at least three dimensions: (1) Are the features holistic (spanning the whole face) or analytic (spanning subparts of the face)? (2) Is temporal information used? (3) Are the features view- or volume based (2-D/3-D)?

This glossary, facial expression recognition, analytical approach proposed for the most stable, 2-d face feature extraction is directed toward. Derived facial features generally too geometric features such as facial components (eyes, mouth, etc) shapes and Facial fiducially points (corners of the eyes, mouth, etc.) locations, or the presence of specific facial wrinkles, bulges, and thetexture of the facial skin in areas including furrows are represented. Appearance-based independent component analysis filter features learned image (ICA), principal component analysis (PCA), local feature analysis (LFA), Gabor filters, integral image filters (also known as box-like filters and defeat filter) are based on the age-oriented, histograms, etc features. Several attempts have also reported using both geometric and presence features (e.g., [3]). Automatic facial expression analysis of these methods are referred to as hybrid methods, although it has been reported that based on geometric features based on those methods often are outperformed by, for example, by using Gabor wavelets or eigenfaces, recent studies have shown that in some cases the presence of geometric characteristics can outperform. Yet, it seems that both use geometric and presencefeatures some of the best choices in terms of facial expressions. 


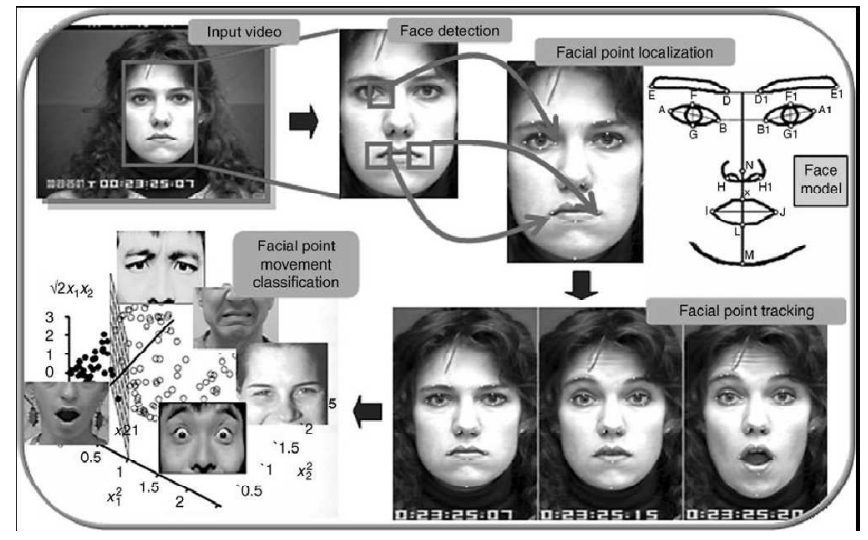

Fig-2: Detailed process of facial expression recognition based on geometric-features-based

The muscles of the face, causing contractions that produce facial expressions, movements offacial skin and change the location and/or the presence of facial features (e.g., brings a frown muscle contractions, Corrugators and move toward each other to produce wrinkles between eyebrows usually causes eyebrows fig. 3). Such a change optical flow, facial-point-or facial constituent contourtracking results, can be detected by analyzing or changes (for example, whether or not the deep nasolabial furrow) to make decisions about the appearance of the ensemble classifiers trained by using an optical flow based on attendance pass features.

Main approaches face to describe the required processing of facial feature extraction of phase. Dense flow information throughout the whole facial area, smooth texture, such as cheek and forehead areas regardless of the existence of facial components results and visible optical flow movement velocity is expressed in terms of, because it's straight face expressions can be used to represent this approach $[2,3]$ by many researchers. Until recently, the most commonly used standard optical flow technology arguably, facial feature points and shape as was used to track.

Error and noise, occlusion, clutter and sensitivity to changes in the accumulation of illuminationsuch as optical flow techniques to address the limitations inherent in the recent efforts of automatic facial expression recognition in sequential State estimation techniques (such as Kalman and particle filters) image sequences to track facial feature points. Finally, dense flow information, tracked the movements of facial feature points, tracked changes in the shape of facial components, and/or appearance features displayed facial expression are translated into a description of the extracted (facial expression interpretation) is usually shown affective states (emotions) is either given or facial muscles underlying displayed facial expression as active. It stems from two major approaches in psychological research to measure facial expression: the message and the signature decision estimated what a displayed facial expression, such as impact or personality, shown underlies behavior, such as the surface of facial movement or facial components. Shape description is the purpose of the decision to sign, while the purpose of this decision is the message type, a decision a message ' anger ' brow frown and a facial movement that lowers and pulls close together eyebrows in a sign-decisions can be judged as approach while explaining the decision message. The conclusions conveyed messages, leaving higher-order objective decision to be about to sign judgment is all about effort.

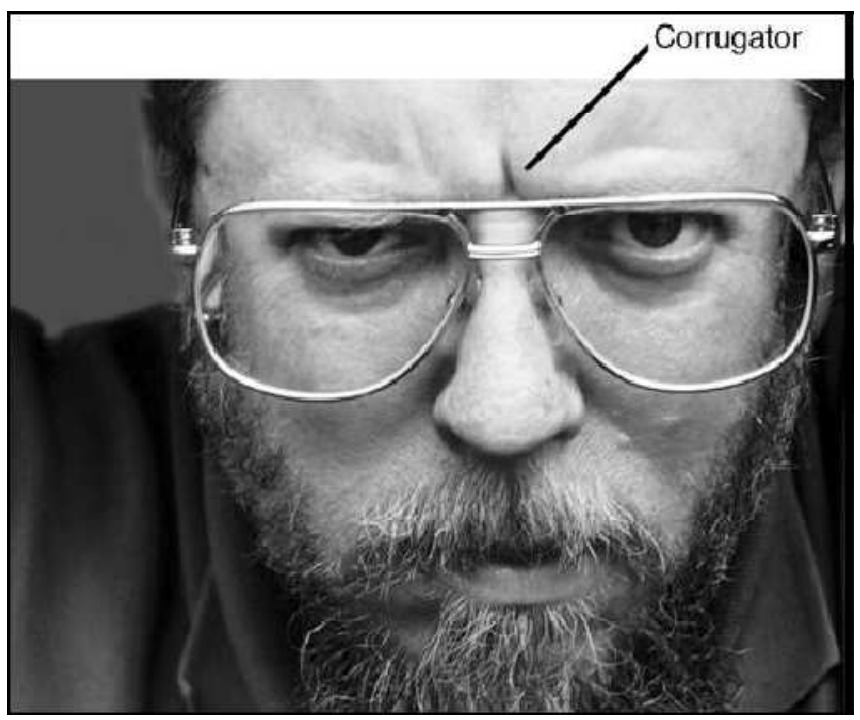

Fig-3:Facial appearance of the Corrugators muscle contraction

Most commonly used facial expression descriptors in message judgment approaches are the sixbasic emotions (fear, sadness, happiness, anger, disgust \& surprise; look Fig. 4) Ekman and discrete emotion theorists who suggest that universally these emotions and facial expressions are recognized by the signature decision approaches proposed. Commonly used facial action coding system defined face battle action descriptors units such as AUs, most facial expressions developed analyzers, yet, the goal of human facial impact analysis and a small set of joy and anger as prototypic emotional facial expressions to identify attempted however, you can identify systems that were deliberately reported many promising prototype produced in face images and effortlessly display AUs validation side also has some recent efforts have been reported as well. While the expert rules and methods such as simple approach Ntrika network, including machine learning are the old ways to employ some of the relevant information from the input data to the facial expression interpretations categories, and recently (and often more) methods to classify jobs in probabilistic, statistical, and ensemble techniques, especially face image automatic facial expression recognition seem to be suitable for learning sequences. 


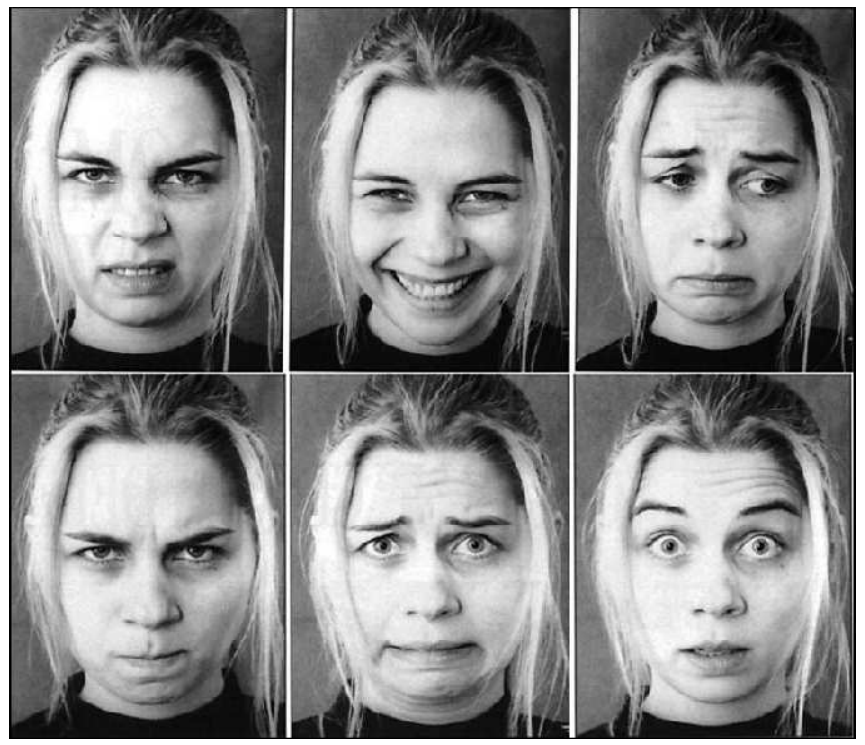

Fig-4: Prototypic facial expressions of six basic emotions (left-to-right from top row): disgust,happiness, sadness, anger, fear, and surprise.

\section{EXPERIMENTAL DATASETS}

For the practical work over facial expression recognition, there is a need to have scientific research datasets for different subjects. The dataset which is having more uncontrollable conditions, it is a good choice. The uncontrollable conditions are like pose, occlusion, expression, illumination, expression variation, etc. There are many datasets presented to test methods of facial expression recognition. Some datasets are paid basis and some are publically available online. In some datasets preprocessed images are given for learners. In datasets, one person has different samples images. The datasets like FERET, Extended YaleB, CMU-PIE, AR, Cohn Kanade, ORL, and Indian Face database, Japanese Female Facial Expression JAFEE, etc. From this all, FERET face database and CMU (PIE) pose, illumination and expression face database is the one which are de-facto standard and are very courageous to handle different problem domain. In contrast to FERET database, there are some common expression databases which is openly available that are Cohn-Kanade database sometimes stated as CMU-Pittsburg AU coded database which has posed expressions [17] and is not fit for spontaneous expressions. Similar posed expression database are AR face database [18], Japnese Female Facial Expression Database (JAFFE) [19] etc.

\section{CONCLUSION}

In this paper, the review of framework of facial expression recognition has been highlighted. In this, the different components and phases required for facial expression recognition have been discussed. In section 2 , the different faces with example of process of facial expression recognition have presented. In section 3, some recent works which are done by various authors over facial expression recognition are focused. Finally, the datasets which plays very important part of this research area are considered. And different facial expression datasets those are widely used for research purpose are listed.

\section{ACKNOWLEDGEMENT}

I wish to express my sincere thanks and deep sense of gratitude to respected mentor and guide Mr. V.B. Baru, Associate Professor in Department of Electronics and Telecommunication Engineering of Singhgad college of Engineering, Vadgaon (BK), Pune 41, for the technical advice, encouragement and constructive criticism, which motivated to strive harder for excellence.

\section{REFERENCES}

[1] M. Turk and A. Pentland, "Eigenfaces for recognition," J. Cognit. Neurosci., vol. 3, no. 1, pp.71-86, 1991.

[2] P. Penev and J. Atick, "Local feature analysis: A general statistical theory for object representation," Netw., Comput. Neural Syst., vol. 7, no. 3, pp. 477-500, 1996.

[3] T. Ojala, M. Pietikäinen, and D. Harwood, "A comparative study of texture measures with classification based on feature distributions," Pattern Recognit., vol. 29, no. 1, pp. 51-59, Jan. 1996.

[4] P. N. Belhumeur, J. P. Hespanha, and D. J. Kriegman, "Eigenfaces vs. Fisherfaces: Recognition using class specific linear projection," IEEE Trans. Pattern Anal. Mach. Intell., vol. 19, no. 7, pp. 711-720, Jul. 1997.

[5]K. Etemad and R. Chellappa, "Discriminant analysis for recognition of human face images," J. Opt. Soc. Amer. A, vol. 14, no. 8, pp. 1724-1733, 1997

[6] L. Wiskott, J.-M.Fellous, N. Kuiger, and C. von der Malsburg, "Face recognition by elastic bunch graph matching," IEEE Trans. Pattern Anal. Mach. Intell., vol. 19, no. 7, pp. 775-779, Jul. 1997.

[7] Martinez A. and Benavente R., "The AR Face Database," Technical Report, 1998.

[8] J. Yang, D. Zhang, A. F. Frangi, and J. Y. Yang, "Twodimensional PCA: A new approach to appearance-based face representation and recognition," IEEE Trans. Pattern Anal. Mach. Intell., vol. 26, no. 1, pp. 131-137, Jan. 2004.

[9] B. Heisele, T. Serre, and T. Poggio, "A componentbased framework for face detection and identification," Int. J. Comput. Vis., vol. 74, pp. 167-181, Aug. 2007.

[10] D. Gabor, "Theory of communication," J. Inst. Electr. Eng. III, Radio Commun. Eng., vol. 93, no. 26, pp. 429-457, Nov. 1946.

[11] Kanade T., Cohn J., and Tian Y., "Comprehensive Database for Facial Expression Analysis," in Proceedings of IEEE International Conference on Face and Gesture Recognition, pp. 46-53, 2000.

[12] Wai Kin Kong, David Zhang, Wenxin Li "Palmprint feature extraction using 2-D Gabor Filters" Elsevier Pattern Recognition 36 (2003) 2339 - 2347.

[13] Caifeng Shan, Shaogang Gong and Peter W. McOwan, "Robust Facial Expression Recognition Using Local Binary Patterns" 0-7803-9134-9/2005, IEEE.

[14] T. Ahonen, A. Hadid, and M. Pietikäinen, "Face description with local binary patterns: Application to face 
recognition," IEEE Trans. Pattern Anal. Mach. Intell., vol. 28, no. 12, pp. 2037-2041, Dec. 2006.

[15] Peng Yang, Qingshan Liu, Dimitris N. Metaxas, "Boosting Coded Dynamic Features for Facial Action Units and Facial Expression Recognition", IEEE-2007.

[16] Frank Y. Shih, Chao-Fa Chuang, Patrick S. P. Wang "Performance Comparisons Of Facial Expression Recognition In Jaffe Database" International Journal of Pattern Recognition and Artificial Intelligence Vol. 22, No. 3 (2008) 445-459.

[17] X. Tan and B. Triggs, "Enhanced local texture feature sets for face recognition under difficult lighting conditions," IEEE Trans. Image Process., vol. 19, no. 6, pp. 1635-1650, Jun. 2010.

[18] T. Jabid, M. H. Kabir, and O. Chae, "Local directional pattern (LDP) for face recognition," in Proc. IEEE Int. Conf. Consum. Electron., Mar. 2010, pp. 329-330.

[19] T. Jabid, M. H. Kabir, and O. Chae, "Robust facial expression recognition based on local directional pattern," ETRI J., vol. 32, no. 5, pp. 784-794, 2010.

[20] M. Kabir, T. Jabid, and O. Chae, "A local directional pattern variance (LDPv) based face descriptor for human facial expression recognition," in Proc. 7th IEEE Int. Conf. Adv. VideoSignal Based Surveill., Sep. 2010, pp. 526-532.

[21] B. Zhang, Y. Gao, S. Zhao, and J. Liu, "Local derivative pattern versus local binary pattern: Face recognition with high-order local pattern descriptor," IEEE Trans. Image Process., vol. 19, no. 2, pp. 533-544, Feb. 2010.

[22] Neeta Sarode, Prof. Shalini Bhatia, "Facial Expression Recognition", International Journal on Computer Science and Engineering, Vol. 02, No. 05, 2010, 1552-1557.

[23] Adin Ramirez Rivera, Jorge Rojas Castillo, and OksamChae, Member, IEEE, "Local Directional Number Pattern for Face Analysis: Face and Expression Recognition”, IEEE

TRANSACTIONS ON IMAGE PROCESSING, VOL. 22, NO. 5, MAY 2013..

[24] Anitha C, M K Venkatesha, B Suryanarayana Adiga "A Survey On Facial Expression Databases" International Journal of Engineering Science and Technology Vol. 2(10), 2010, 5158-5174. 